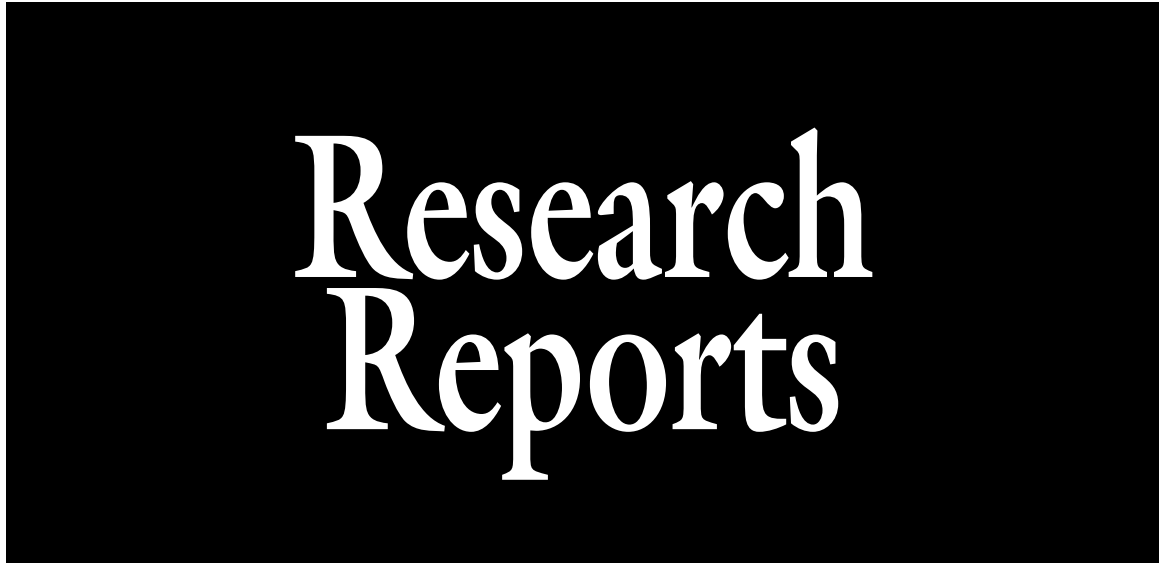

\title{
An Evaluation of Scent-discriminating Canines for Rapid Response to Agricultural Diseases
}

\author{
Julian Mendel ${ }^{1,2,3}$, Kenneth G. Furton ${ }^{2,3}$, and DeEtta Mills ${ }^{1,2,3,4}$
}

AdDITIONAL INDEX wORDs. early detection, disease management, laurel wilt disease, avocado, fruit crops, fungal pathogen

SUMMARY. Laurel wilt disease, incited by Raffaelea lauricola, has resulted in the death of more than $\mathbf{3 0 0}$ million laurel trees (Lauraceae) in the United States. One such tree is the commercially important avocado (Persea americana), the second largest tree crop in Florida other than citrus (Citrus sp.). This disease affects the industry in South Florida and two larger avocado industries in Mexico and California have taken notice. Trees succumb soon after infection, and once external symptoms are evident, the disease is very difficult to control and contain as the pathogen can spread to adjacent trees via root grafting. Presently, there is no viable, cost-effective method of early diagnosis and treatment. This study was undertaken to evaluate the use of scent-discriminating canines (Canis familiaris) for the detection of laurel wilt-affected wood from avocado trees. Three canines, one Belgian Malinois and two Dutch Shepherds, were trained and studied for this ability. In addition, prevailing weather conditions were recorded and evaluated to determine their effect on canine performance. The results of this evaluation indicated that canines can detect laurel wilt-affected wood and the laurel wilt pathogen and may be useful in the detection of laurel wilt-diseased trees in commercial groves.

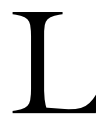

aurel wilt is the consequence of an invasive species, the redbay ambrosia beetle (RAB) (Xyleborus glabratus) introduced into the United States in untreated wooden packaging material. It was first detected in diseased redbay trees (Persea borbonia) in Savannah, GA, in 2002 (Mayfield and Thomas, 2006). The beetle, originally from Asia, was found to be the vector of the disease in redbay trees and was thus named RAB (Fraedrich et al., 2008; Hanula et al., 2008; Mayfield and Thomas, 2006). The term ambrosia refers to an ecological relationship that these beetles share with fungal partners. These partners have coevolved to become dependent on one another for survival and dispersal. Ambrosia beetles belong to the subfamily Scolytinae and are some of the most common and the most devastating pests to plants known to date (Ploetz et al., 2013). Although its original introduction was through the $\mathrm{RAB}$, it is important to note that in avocado, multiple beetle vectors have demonstrated the ability either as a contaminant or a new vector to inoculate trees (Carrillo et al., 2014).
Ambrosia beetles typically harbor fungal spores within specialized sacs known as mycangia, which excrete fungal spores as they bore into host trees, thus inoculating the tree with the fungus. The beetle then actively cultivates or farms the fungal gardens within excavated galleries as a food source for itself and its developing larvae (Batra, 1967; Ploetz et al., 2013). In laurel wilt disease, this particular fungal partner was identified as a lethal pathogen $R$. lauricola (Fraedrich et al., 2008; Harrington et al., 2008). The name, laurel wilt disease, was coined when the fungal spores of $R$. lauricola were shown to infect and lead to wilt symptoms in many species of laurel trees (Harrington et al., 2008), including the commercially important avocado (Ploetz et al., 2017a). This is a systemic disease incited by the clogging of the xylem vessels through the tree's production of tylose-, phenolic-, pectin-, and lipid-containing gums or gels as it tries to defend itself from the systemic infection (Inch et al., 2012), a general response that can be produced because of several host insults. The result of blocked xylem vessels is compromised water and nutrient flow during evapotranspiration, visible wilting of the leaves, tissue necrosis, and, ultimately, the death of the tree. The presence of the fungus can be indicated visually through black/brown-stained sapwood, caused by the host response (Fraedrich et al., 2008; Inch and Ploetz, 2012; Inch et al., 2012).

Laurel wilt disease is highly aggressive and it has been demonstrated that only a few spores (as low as 100 conidia) or a single beetle-boring event can be sufficient to elicit the systemic disease in avocado. More than 300 million wild laurels have been lost and presently laurel wilt disease is rapidly spreading through the South Florida avocado groves, a \$54-million/year industry (Evans et al., 2010), with the cost of replacement of trees rising to $\$ 400$ million (Evans et al., 2010). This

\begin{tabular}{llll}
\hline $\begin{array}{l}\text { Units } \\
\begin{array}{l}\text { To convert U.S. to SI, } \\
\text { multiply by }\end{array}\end{array}$ & U.S. unit & SI unit & $\begin{array}{l}\text { To convert SI to U.S., } \\
\text { multiply by }\end{array}$ \\
\hline 0.0929 & $\mathrm{ft}^{2}$ & $\mathrm{~m}^{2}$ & 10.7639 \\
1.6093 & $\mathrm{mph}$ & $\mathrm{km} \cdot \mathrm{h}^{-1}$ & 0.6214 \\
28.3495 & $\mathrm{OZ}$ & $\mathrm{g}$ & 0.0353 \\
$\left({ }^{\circ} \mathrm{F}-32\right) \div 1.8$ & ${ }^{\circ} \mathrm{F}$ & ${ }^{\circ} \mathrm{C}$ & $\left({ }^{\circ} \mathrm{C} \times 1.8\right)+32$
\end{tabular}


economic and ecological disaster will be even greater if this disease infects the larger avocado production areas such as California and Mexico.

Presently, the fungicide propiconazole (TILT ${ }^{\circledR}$; Syngenta Crop Protection, Wilmington, DE) that suppresses the growth of the fungus but does not kill it is used to protect trees from infection. This formulation can provide 11-12 months of protection depending on the method of administration into the trees (Ploetz et al., 2017a, $2017 b, 2017 \mathrm{c}$ ). However, to prophylactically treat all trees comes with a high cost that many small farmers simply cannot afford. Studies geared toward early detection of laurel wilt disease using aerial imagine and remote sensing with drones have demonstrated promise in distinguishing between laurel wilt-affected trees from healthy trees as well as other stressors (de Castro et al., 2015a, 2015b). To aid in the early detection and control of this disease, scent-discriminating canines have been trained on laurel wilt-affected avocado wood to enable earlier detection and a more focused hot spot treatment that provides a cost-effective treatment plan for farmers.

Presently, canines are extensively used in law enforcement and forensics in the location of missing people, explosives, drugs, weapons, and ammunition (Furton and Myers, 2001). Furthermore, canines have

This work was originally an oral presentation at the Annual Meeting of the Florida State Horticultural Society under the title: "Disease, Dogs and Drones." The Florida Department of Agriculture and Consumer Services (FDACS) supported this research through a Specialty Crop Block Grant (\#020731) to DeEtta Mills. Julian Mendel was supported in part during this research through the MBRS-RISE program (NIH/NIGMS R25 GM061347). The presented content is solely the responsibility of the authors and does not necessarily represent the official views of the National Institutes of Health.

We thank Innovative Detection Concepts (iDC) for providing the canines used in this project, as well as canine trainers, Peter Nunez and Lourdes Eldin, for training and handling the canines. A special thanks also goes to Jennifer Gebelein and Carlos Pulido, as well as Alison Simon.

${ }^{1}$ Department of Biological Sciences, Florida International University, OE 167, 11200 SW 8th Street, Miami, FL 33199

${ }^{2}$ Department of Chemistry and Biochemistry, Florida International University, CP 302,11200 SW $8^{\text {th }}$ Street, Miami, FL 33199

${ }^{3}$ International Forensic Research Institute, Florida International University, OE 116, 11200 SW $8^{\text {th }}$ Street, Miami, FL 33199

${ }^{4}$ Corresponding author. E-mail: millsd@fiu.edu.

https://doi.org/10.21273/HORTTECH03794-17 demonstrated the ability to detect invasive species of spotted knapweed [Centaurea stoebe (Goodwin et al., 2010)], brown tree snake [Boiga irregularis (Savidge et al., 2011)], desert tortoise [Gopherus agassizii (Cablk and Heaton, 2006; Nussear (Godfrey, 2014; McCulloch et al., 2006; Moser and McCulloch, 2010). They have even been used to detect bed bugs (Cimex lectularius), termites (Reticulitermes sp., Coptotermes sp., Cryptotermes sp., and Inscisitermes sp.), and mold (Aspergillus sp., Penicillium sp., and Stachybotrys sp.). The highly sensitive canine olfactory system, roughly 10,000-100,000 times more sensitive than humans, is capable of detecting odor concentrations at 1-2 parts/trillion. This is attributed to the size of the organ, density of neurons, the number of functional receptors, and the physical anatomy of the olfactory system (Craven et al., 2009). This study represents the first of its kind, to the author's knowledge, evaluating the use of canine odor discrimination in the early detection of this plant disease.

\section{Materials and methods Study location}

This study was conducted in a privately owned mango (Mangifera sp.) grove in the Redland agricultural district near Homestead, FL, under the Institutional Animal Care and Use Committee (IACUC) approval et al., 2008)], and various cancers

(IACUC-15-011-CR01). No laurel wilt was detected in the immediate vicinity.

\section{Canine selection and training aids}

Canines with no prior training were acquired through the United States K-9 Academy and Police Dog Training Center in Hialeah, FL (Fig. 1), and trainers and handlers were provided with training aids. The training aids consist of laurel wilt-affected avocado wood as well as the fungal pathogen $R$. lauricola placed in controlled odor mimic permeation system [COMPS (Furton and Harper, 2008)]. COMPS are specialized polymer bags that allow odor to escape at a constant rate but contain the biological or chemical agent within (Fig. 2). The amount of plant material used was $\approx 15-20 \mathrm{~g}$, was sealed within COMPS, stored in re-sealable aluminum envelopes and allowed to equilibrate at least $24 \mathrm{~h}$ before use.

\section{Canine training}

The training of the canines took place in three main phases.

Phase 1. Using an open outdoor area of "tall grasses," the dogs were evaluated for their "hunt drive." This phase involved simple fetch exercises with a chew toy to determine whether they had the desire to search for extended periods of time for the reward of playing with the toy.

Phase 2. This phase involved the use of stainless steel odor boxes and a universal detector calibrant [UDC

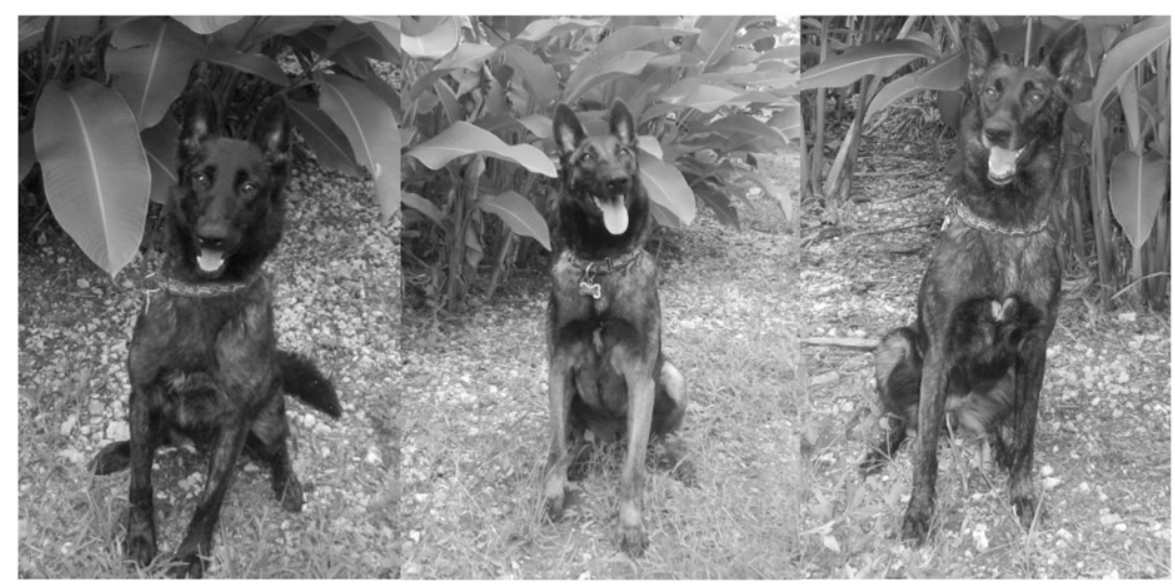

Fig. 1. The three canines used in the present study that have been trained to detect laurel wilt, from left to right: Candy, Cobra, and OneBetta. Candy and OneBetta are Dutch Shepherds, whereas Cobra is a Belgian Malinois. These canines were trained to seek out and identify presymptomatic trees affected by laurel wilt before any visible symptoms of the disease were visible. 


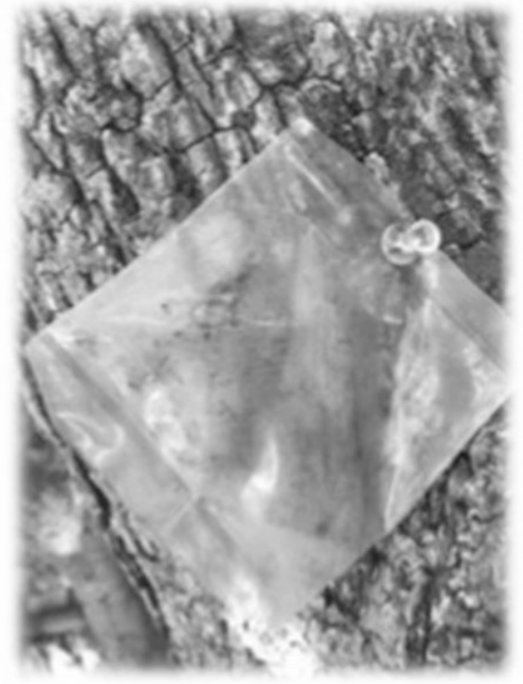

Fig. 2. The controlled odor mimic permeation system (COMPS) used to encase the materials used for training canines to detect laurel wilt-infected avocado wood. COMPS allow for a uniform release of the volatiles associated with the sample within, but are able to safely contain the biological agent within. The aid shown has laurel wilt-infected avocado wood sealed inside.

(Furton and Beltz, 2017)], which is a synthetic compound with an odor found not to occur in nature. Here, the goal was to initiate odor association with the process of rewarding canines with the chew toy.

Phase 3. This phase involved the substitution of the UDC with the odor of interest or target odor. In this case, laurel wilt-infected avocado wood, or pure cultures of $R$. lauricola, was used. This initial training process was completed in $\approx 1$ month followed by reinforcement training a minimum of three times/week thereafter; canines were monitored and evaluated over the course of 1 year and 229 training sessions.

\section{Evaluation of training success}

Canine performance was evaluated within an $\approx 2000-\mathrm{ft}^{2}$ planting of mango trees to ensure that $R$. lauricolainfected avocado trees would not be present in the training site. The time in seconds from handler release to training aid location and alert (sitting at the base of the tree in which the target odor was detected) was recorded. In addition to these data, the number of false alerts, failures to alert, and other observations regarding environmental and other distractors that seemed to affect the canine's accuracy in an agricultural setting were recorded.

\section{Evaluating the effect of weather conditions on canine performance}

Temperature, relative humidity, and wind speed were recorded at the start of each canine deployment using a weather monitor (Kestrel 4500; Loftopia, Birmingham MI).

\section{3-D controlled study}

A study was conducted to evaluate whether canine performance was influenced when both the handler and the canine were blind to the placement of the training aids (simulation of a real deployment scenario). Within the same mango grove, a training area consisting of 25-30 trees was designated and each tree was assigned a number. COMPS training aids with healthy or laurel wilt-affected avocado wood were prepared and the selection of trees in which to hide/ place the training aids was performed using a random number generator. In each run, three positive aids and one negative control (healthy wood) were used. The evaluator then walked up and down each row, stopping at and touching each tree to ensure that the canines did not track the human scent but rather were searching for the training aid. After $5 \mathrm{~min}$, the handler teams worked with their canines to locate the hidden aids, and the time to alert on a positive aid was recorded. On each of the three different days, training aid detection was evaluated at 9:00 AM, 1:00 PM, and 4:00 PM, with new random placements of the training aids for each run.

\section{Pathogen detection and negative control study}

Training aids with only the laurel wilt pathogen, grown in pure culture and obtained through University for Florida Tropical Research and Education Center (UF-TREC) pathology laboratory (Ploetz et al., 2011), were placed in sealed COMPS bags to determine if the canines were able to successfully detect and alert to just the fungus. All materials such as empty COMPS bags as well as the media used to grow the pathogen were also set out as distractors during a 3-d trial (conducted at a different time to the previously described 3 -d trial). A "positive pathogen" aid was placed within the training area, as well as empty COMPS bags and one growth media-only sample sealed in a COMPS. The dogs were then deployed, and observations were made and alerts were recorded.

\section{Canine/handler certification}

Performance of the canine handler teams was evaluated with, independent evaluators (not otherwise involved with the study) following the approved best practice guidelines of the Scientific Working Group on Dogs and Orthogonal Detector Guidelines (SWGDOG), which specify that dogs respond with a minimum of $90 \%$ positive rate and a corresponding maximum false alert rate of $10 \%$ (Furton et al., 2010).

\section{Results}

Training evaluation. A total of 229 training sessions with three canines, Cobra (73), OneBetta (78), and Candy (78), were performed and the search-locate-alert time for canines was recorded. The averaged time to alert was $40.82,37.43$, and $48.50 \mathrm{~s}$ for Cobra, OneBetta, and Candy, respectively. There was no significant difference between the times between canines [analysis of variance (ANOVA) $P>0.05, \mathrm{df}=2$, $F=2.04]$. There were a total of 12 false alerts in which the canines alerted to a tree not holding a positive training aid, 10 of which were attributed to OneBetta. There were also 12 failures to alert recorded, in which the canines did not locate the positive training aid during the session, with 10 being attributed to Candy. To evaluate canine performance, the accuracy (ACC) and positive predictive value (PPV) for each canine was calculated. The average ACC and PPV were determined to be $99.4 \%$ and $94.8 \%$, respectively (J. Mendel, C. Burns, B. Kallifatidis, E. Evans, J. Crane, K.G. Furton, and D. Mills, unpublished data).

EFFECT OF WEATHER ON PERFORMANCE. Scatterplots with trend lines (Figs. 3-5) demonstrated shorter times to target location associated with temperature and wind speed, compared with longer search times associated with higher humidity. Multiple linear regression analysis revealed an $R^{2}$ of 0.07 , with the only significant variable being temperature $(P<0.05)$. 


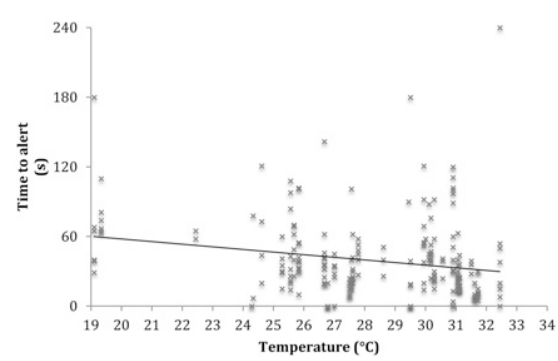

Fig. 3. The relationship between temperature and the time to alert to the training aid over the course of 229 training sessions with the three canines trained to detect laurel wiltinfected avocado wood. The trend indicated that increasing environmental temperatures lead to decreased search times $(P<0.05)$; $\left(1.8 \times{ }^{\circ} \mathrm{C}\right)+32={ }^{\circ} \mathrm{F}$.

It was observed during the study that false alerts were most often observed on trees downwind but near to the target tree. The analyses found that, with the exception of two alerts, false alerts were all observed when wind speeds were about $5 \mathrm{~km} \cdot \mathrm{h}^{-1}$ or higher (Fig. 6).

Controlled studies. A 3-d controlled study demonstrated high accuracy and precision by all three canines, and ANOVA results showed no difference in the time to alert during different times of the day for deployment $[P>0.05, \mathrm{df}=2, F=3.47$ (Table 1)]. When compared with the 229 canine training sessions, it was observed that the randomized, double-blind study led to significantly longer search times $[P<0.05, \mathrm{df}=1, F=5.10$ (Table 1)]. The second controlled study involving a variety of negative controls led to only one false-positive alert on growth media by OneBetta (Table 2).

\section{Discussion}

This study demonstrated the ability of canines to detect laurel wilt. The goals of this study were to evaluate the success of canine training over an extended period, during the time period of Sept. 2014-July 2015 in Homestead, FL. With an average time to locate a positive sample of affected avocado wood that ranged between $\approx 37-49$ s with the canines working with $\approx 25-30$ trees in the study area, they proved to be not only an accurate detection option but also a truly rapid one. The high accuracy observed in this study was comparable to a study involving the detection of tethered tortoises (Cablk and Heaton, 2006),

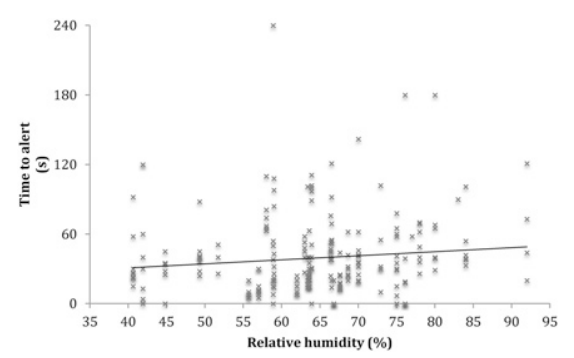

Fig. 4. The relationship between relative humidity and the time to alert to the training aids, which contained laurel wilt-infected avocado wood that the canines were trained to detect, over the course of 229 training sessions with three canines where increasing humidity was associated with slightly longer search times (not significant, $P>0.05$ ), yet the canine's performance was not affected by higher humidity as observed in the study.

which boasted $>90 \%$ accuracy in locating both surface and burrowed animals. Another study, with the same species of desert tortoise, evaluated detection accuracy in the wild with free-roaming tortoises rather than those tethered to a specific location. They reported $70 \%$ accuracy (14).

A low number of false alerts, only 12, were detected and interestingly, OneBetta was the canine responsible for 10 of those 12 alerts. This highlights an important point that each animal's behavior differs. It was noted by the handler and the trainer that OneBetta has a tendency to be eager to alert as soon as she picked up the odor cone, but on denial of her reward, she then moves on to locate the point source. Failures to locate a training aid during a deployment were also noted. A large number of these failed deployments, 11 of them, were due to fallen fruit in the grove, which distracted the canines and resulted in long and interrupted search times and were early in their training. Other distractions that resulted in longer times and failed deployments included the presence of other wild animals being flushed from the grasses that distracted the canines from their searches. Fine-tuning of obedience training proved to reduce these incidents over the course of the training but highlights important factors to consider when deploying canines in an agricultural or uncontrolled environment. In Guam, a study involving the location of brown

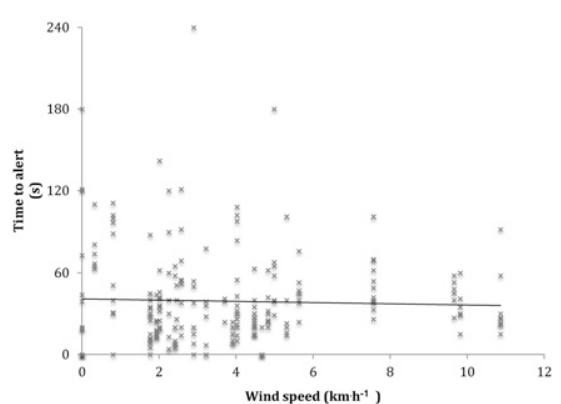

Fig. 5. There was no significant relationship $(P>0.05)$ between wind speed and the time to alert to the laurel wilt training aids over the course of 229 training sessions with three canines. The data indicated that increasing wind speed did not significantly affect canine performance or their ability to locate target odors; however, most false alerts were recorded at wind speeds greater than $48 \mathrm{~km} \cdot \mathrm{h}^{-1} ; 1 \mathrm{~km} \cdot \mathrm{h}^{-1}=$ $0.6214 \mathrm{mph}$.

tree snakes, reported a low 35\% success accuracy for the canine teams (Savidge et al., 2011). Perhaps, the lower accuracy in that study can be attributed to the added difficulty in locating moving targets. Another study involved the detection and location of an invasive plant species of knapweed. This small plant can be difficult to locate in bushy understory. The study made a direct comparison between handler canine teams and human-only surveyors (Goodwin et al., 2010). An overall canine accuracy of $81 \%$ was observed and canine teams outperformed human-only teams in their ability to locate the elusive plant.

The present study also calculated the PPV or precision of canines in their detection of laurel wilt-diseased avocado wood. The results ranged between $87 \%$ and $100 \%$ for the three canines. The high accuracy and precision in more than 229 deployments coupled with the design of the training, using infected avocado wood and training in an agricultural setting, strongly supports the viability of using canine detectors for laurel wilt disease detection.

The results of the controlled study in which both handlers and canines were blind to the placement of training aids had an average time to alert of about $58 \mathrm{~s}$ which was found to be significantly higher, using a Student's $t$ test $(P<0.05)$, than the average of the 229 previous canine 


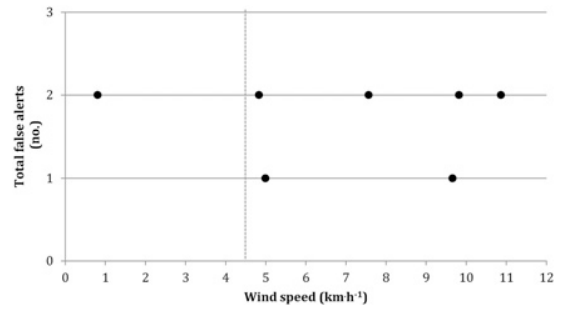

Fig. 6. The relationship between total number of false alerts with increasing wind speed. With the exception of two false alerts by OneBetta at $\approx 0.8$ $\mathrm{km} \cdot \mathrm{h}^{-1}$, all false alerts came when wind speed was $\approx 4.8 \mathrm{~km} \cdot \mathrm{h}^{-1}$ and higher. These data demonstrated the potential impact on canine performance through increased occurrence of false alerts due to the movement or disturbance of the target odor, laurel wilt-infected avocado wood, that the canines were trained to detect from the true source to other trees nearby because of high winds; $1 \mathrm{~km} \cdot \mathrm{h}^{-1}=0.6214 \mathrm{mph}$. deployments (about $42 \mathrm{~s}$ ). However, because of the substantially smaller number of deployments in this experiment, it is unclear whether this was truly due to the additional controls and precautions being taken to mask the human trail or whether the difference would be significant with a comparable number of trials. During the controlled study, no alerts to healthy avocado wood COMPS were observed and in addition, no false alerts were observed to the blank COMPS or other distractors. There was no significant difference found using ANOVA between the accuracy of the three canines or in deployments taking place at different times of the day $(P>0.05)$.

Another goal of this study was to investigate the effect, if any, on canine performance in the often-harsh conditions such as high temperatures and humidity in South Florida's agricultural

Table 1. A summary for the controlled 3-d evaluations of canine performance in locating laurel wilt training aids. Analysis of variance results indicated no significant difference between canines as well as no significant difference between time of day $(P>0.05, \mathrm{df}=2, F=3.47)$ with respect to time to alert (T1, T2, and T3). However, comparing these results to the 229 routine training results described in the text showed a significantly increased time to alert when both the handlers and canines are blind to the location of the training aid $(P<0.05, \mathrm{df}=1$, $F=5.10)$.

\begin{tabular}{lccccr}
\hline Canine name & Training time & T1 $(\mathrm{s})$ & T2 $(\mathrm{s})$ & T3 (s) & Avg $(\mathrm{s})^{\mathrm{y}}$ \\
\hline Candy & D1- 9:00 AM & 43.54 & 45.01 & 71.2 & 53.25 \\
Candy & D2- 1:00 PM & 60 & 50 & 43 & 51.00 \\
Candy & D3- 4:00 PM & 150 & 25 & 32.9 & 69.30 \\
Cobra & D1- 9:00 AM & 85 & 63 & 169 & 105.67 \\
Cobra & D2- 1:00 PM & 40 & 43 & 80 & 54.33 \\
Cobra & D3- 4:00 PM & 3.43 & 27.27 & 14.68 & 15.13 \\
OneBetta & D1- 9:00 AM & 77 & 7.36 & 40.6 & 41.65 \\
OneBetta & D2- 1:00 PM & 53 & 17 & 80 & 50.00 \\
OneBetta & D3- 4:00 PM & 120 & 51 & 71 & 80.67 \\
\hline
\end{tabular}

${ }^{\mathrm{Z}} \mathrm{D} 1, \mathrm{D} 2$, and $\mathrm{D} 3$ represent days 1,2 , and 3 . On each day, canines were evaluated at three different times per day for the time taken to locate three training aids (Tl-T3).

${ }^{y}$ In each case, healthy avocado wood was used as a negative control and no alerts were observed.

environments. A previous study by Nussear et al. (2008) examined whether the prevailing weather conditions (wind speed, temperature, and relative humidity) affected the search times in canine handler teams in locating desert tortoises and found no relationship. Savidge et al. (2011) in the detection of snakes demonstrated increased success with decreasing wind speeds and increasing humidity. In an attempt to evaluate the predictive power of these variables on the time to alert, a linear regression analysis was done and demonstrated a weak model with an $R^{2}$ value of 0.07 ; however, temperature had a significant probability value (ANOVA) indicating that it possibly has a greater effect on odor dispersal from COMPS allowing for faster detection times. It is known that high temperatures can adversely affect canines (Furton et al., 2010), so perhaps, the small search area combined with higher temperatures led to greater odor dispersal and thus the faster detections observed. It is clear, however, that the canines are capable of performing at high levels under all the conditions observed during the course of the study. Interestingly it was observed that a higher number of false alerts were seen at higher wind speeds [ $>4.8 \mathrm{~km} \cdot \mathrm{h}^{-1}$ (Fig. 6)], particularly for OneBetta who was responsible for 10 of the 12 false alerts over the course of the study. False alerts were usually on a tree downwind of the target tree. Only two false alerts were observed at low wind speeds. It is known that wind can transport odors making higher winds ideal for tracking and location over long distances (Cablk and Heaton, 2006); however, it can also result in pooling of odors away from the source and dispersal can make it difficult for the

Table 2. The results of a 2-week trial with the laurel wilt pathogen cultures and negative control training aid materials for two canines, Cobra and OneBetta. This trial evaluated the ability of the canines to discriminate nontarget odors from the target odor. Each training aid type was stored in controlled odor permeations systems (COMPS). Treatments and corresponding responses by each canine (Alert/No Alert) are shown.

\begin{tabular}{|c|c|c|c|c|c|c|}
\hline Day & Canine name & Pathogen + malt agarz & Empty COMPS & Empty COMPS & Empty COMPS & Malt agar only \\
\hline 1 & Cobra & Alert & No Alert & No Alert & No Alert & No Alert \\
\hline 1 & OneBetta & Alert & No Alert & No Alert & No Alert & Alert \\
\hline 2 & Cobra & Alert & No Alert & No Alert & No Alert & No Alert \\
\hline 2 & OneBetta & Alert & No Alert & No Alert & No Alert & No Alert \\
\hline 3 & Cobra & Alert & No Alert & No Alert & No Alert & No Alert \\
\hline 3 & OneBetta & Alert & No Alert & No Alert & No Alert & No Alert \\
\hline
\end{tabular}

${ }^{\mathrm{z}}$ COMPS with the pathogen grown on malt agar.

${ }^{y}$ COMPS bag without any pathogen content.

${ }^{x}$ Pure malt agar sealed in a COMP bag with no microbial growth. 
canines to pinpoint targets (Savidge et al., 2011). Canine performance was unaffected by climate conditions in a previous study with desert tortoises (Nussear et al., 2008) whereas, with respect to brown tree snakes in Guam, increasing humidity had a positive effect with performance, and lower wind speeds increased the success (Savidge et al., 2011).

Last, the canines after being presented with the laurel wilt pathogen, grown in pure culture, successfully demonstrated the ability to detect and alert to only the fungus (Table 2). The fact that the canines were able to alert to and detect the pathogen in laboratory-grown cultures, when they were primarily trained on the entire scent picture of the pathogen growing within avocado wood, is a testament to their specificity. This is not surprising as previous studies demonstrated this ability with canines trained on toxic mold species growing on wood (Griffith et al., 2007). The study on mold aimed to determine whether through the use of analytical chemistry, they could identify volatile signature differences between closely related species of mold and whether detector dogs could distinguish between nontoxic and toxic species. Whereas canines demonstrated $>90 \%$ accuracy on toxic mold growing on wood, this translated to $\approx 70 \%$ with laboratory-grown cultures (Griffith et al., 2007). This study also discussed the usefulness of analytical techniques to identify unique chemical signatures to improve training through more specific odor mimics as aids, and has also been investigated with explosives and drugs (Harper et al., 2005). It is also important to note that closely related species of fungi exist to the pathogen used in this study and it is unclear whether the canines would alert to potentially similar odors. The use of additional cultures of different species including those isolated from avocado trees is underway and will be evaluated in future studies.

Another interesting study by Waggoner et al. (1998), discussed the effect of extraneous odors on the ability of canines to detect target odors. This study involved the masking of the target with increasing concentrations of nontarget odors (Waggoner et al., 1998). This is significant as most training takes place in controlled environments indoors with no extraneous odors. The results demonstrated a remarkable ability to alert correctly to the target in the presence of masking odors, but that in extremely high nontarget odor environments, their detection capabilities can be reduced (Waggoner et al., 1998). In the present study, the method of training in outdoor agricultural settings was well thought out to enable the canines to be accustomed to as many possible interfering odors as possible as they would experience them in their intended deployment application. This study demonstrated successful training, usefulness, and evaluation of the detection capabilities of scent-discriminating canines and provides a new tool in the arsenal in defense against plant diseases in an agricultural or outdoor, uncontrolled environment.

\section{Conclusions}

This study demonstrated the ability of scent-discriminating canines to locate laurel wilt-diseased avocado wood with high accuracy and speed. During the course of the study, 229 trials were performed, and only 12 false alerts were observed and 12 failures to alert. It was observed that canines are capable of high levels of performance even in harsh weather conditions such as high heat and humidity.

This study provided the proof of principle that canines can detect agricultural diseases such as laurel wilt and can be a powerful management tool if the disease is caught in its earliest stages. Laurel wilt disease is especially difficult and costly to manage. Once symptoms of this disease become visible to humans, it is already too late for treatment and that the tree must be removed as it is highly likely that the pathogen has spread to adjacent trees, through root grafts. Analogous to early cancer detection in humans (Godfrey, 2014; McCulloch et al., 2006; Moser and McCulloch, 2010), the ability to detect trees at an earlier disease stage would provide a significant advantage in the battle to stop the spread of this deadly crop disease.

\section{Literature cited}

Batra, L.R. 1967. Ambrosia fungi: A taxonomic revision, and nutritional studies of some species. Mycologia 59(6):9761017.

Cablk, M.E. and J.S. Heaton. 2006. Accuracy and reliability of dogs in surveying for desert tortoise (Gopherus agassizii). Ecol. Appl. 16(5):1926-1935.

Carrillo, D., R.E. Duncan, J.N. Ploetz, A.F. Campbell, R.C. Ploetz, and J.E. Peña. 2014. Lateral transfer of a phytopathogenic symbiont among native and exotic ambrosia beetles. Plant Pathol. 63(1): 54-62.

Craven, B.A., E.G. Paterson, and G.S. Settles. 2009. The fluid dynamics of canine olfaction: Unique nasal airflow patterns as an explanation of macrosmia. J. R. Soc. Interface 7(47):933-943.

de Castro, A.I., R. Ehsani, R. Ploetz, J.H. Crane, and J. Abdulridha. 2015a. Optimum spectral and geometric parameters for early detection of laurel wilt disease in avocado. Remote Sens. Environ. 171: 33-44.

de Castro, A.I., R. Ehsani, R.C. Ploetz, J.H. Crane, and S. Buchanon. 2015b. Detection of laurel wilt disease in avocado using low altitude aerial imaging. PLoS One 10(4):e0124642.

Evans, E.A., J. Crane, A. Hodges, and J.L. Osborne. 2010. Potential economic impact of laurel wilt disease on the Florida avocado industry. HortTechnology 20: 234-238.

Fraedrich, S.W., T.C. Harrington, R.J. Rabaglia, M.D. Ulyshen, A.E. Mayfield, III., J.L. Hanula, J.M. Eikwort, and D.R. Miller. 2008. A fungal symbiont of the redbay ambrosia beetle causes a lethal wilt in redbay and other Lauraceae in the southeastern United States. Plant Dis. 92(2):215-224.

Furton, K.G. and K. Beltz. 2017. Universal detector calibrant. U.S. Patent 9575038. Filed 19 Jan. 2016. Issued 21 Feb. 2017.

Furton, K.G, J. Greb, and H. Holness. 2010. The scientific working group on dog and orthogonal detector guidelines (SWGDOG). Natl. Criminal Justice Reference Serv. 155, U.S. Dept. Justice, Rockville, MD.

Furton, K.G. and R.J. Harper. 2008. Controlled odor mimic permeation system. U.S. Patent 9706755 . Filed 18 July 2007. Issued 18 July 2008.

Furton, K.G. and L.J. Myers. 2001. The scientific foundation and efficacy of the use of canines as chemical detectors for explosives. Talanta 54(3):487-500.

Godfrey, A. 2014. Canine scent detection of human cancers: Is this a viable technique for detection? Veterinary Nursing J. 29(12):392-394.

Goodwin, K.M., R.E. Engel, and D.K. Weaver. 2010. Trained dogs outperform human surveyors in the detection of rare 
spotted knapweed (Centaurea stoebe). Invasive Plant Sci. Mgt. 3(2):113-121.

Griffith, R.T., K. Jayachandran, W. Whitstine, and K.G. Furton. 2007. Differentiation of toxic molds via headspace SPME-GC/MS and canine detection. Sensors (Basel) 7(8):1496-1508.

Hanula, J.L., A.E. Mayfield, S.W. Fraedrich, and R.J. Rabaglia. 2008. Biology and host associations of redbay ambrosia beetle (Coleoptera: Curculionidae: Scolytinae), exotic vector of laurel wilt killing redbay trees in the southeastern United States. J. Econ. Entomol. 101(4):1276-1286.

Harper, R.J., J.R. Almirall, and K.G. Furton. 2005. Identification of dominant odor chemicals emanating from explosives for use in developing optimal training aid combinations and mimics for canine detection. Talanta 67(2):313-327.

Harrington, T.C., S.W. Fraedrich, and D.N. Aghayeva. 2008. Raffaelea lauricola, a new ambrosia beetle symbiont and pathogen on the Lauraceae. Mycotaxon 104(2):399-404.

Inch, S.A. and R.C. Ploetz. 2012. Impact of laurel wilt, caused by Raffaelea lauricola, on xylem function in avocado, Persea americana. For. Pathol. 42(3):239-245.

Inch, S.A., R.C. Ploetz, B. Held, and R. Blanchette. 2012. Histological and anatomical responses in avocado, Persea americana, induced by the vascular wilt pathogen, Raffaelea lauricola. Botany 90(7):627-635.

Mayfield, III, A.E., and M.C. Thomas. 2006. The redbay ambrosia beetle, Xyleborus glabratus Eichhoff (Scolytinae: Curculionidae). Pest Alert, Florida Dept. Agr. Consumer Serv., Tallahassee, FL.

McCulloch, M., T. Jezierski, M. Broffman, A. Hubbard, K. Turner, and T. Janecki. 2006. Diagnostic accuracy of canine scent detection in early-and late-stage lung and breast cancers. Integr. Cancer Ther. 5(1):30-39.

Moser, E. and M. McCulloch. 2010. Canine scent detection of human cancers: A review of methods and accuracy. J. Vet. Behav. 5(3):145-152.

Nussear, K.E., T.C. Esque, J.S. Heaton, M.E. Cablk, K.K. Drake, C. Valentin, J.L. Lee, and P.A. Medica. 2008. Are wildlife detector dogs or people better at finding desert tortoises (Gopherus agassizii)? Herpetol. Conserv. Biol. 3(1):103-115.

Ploetz, R.C., M.A. Hughes, P.E. Kendra, S.W. Fraedrich, D. Carrillo, L.L. Stelinski, J. Hulcr, A.E. Mayfield, III., T.L. Dreaden, J.H. Crane, E.A. Evans, B.A. Schaffer, and J. Rollins. 2017c. Recovery plan for laurel wilt of avocado, caused by Raffaelea lauricola. Plant Health Prog. 18(2):51-77.
Ploetz, R.C., J. Hulcr, M.J. Wingfield, and Z.W. de Beer. 2013. Destructive tree diseases associated with ambrosia and bark beetles: Black swan events in tree pathology? Plant Dis. 97(7):856-872.

Ploetz, R.C., P.E. Kendra, R.A. Choudhury, J. Rollins, A. Campbell, K. Garrett, M. Hughes, and T. Dreaden. 2017a. Laurel wilt in native and agricultural ecosystems: Understanding the drivers and scales of complex pathosystems. Forests 8(2):48.

Ploetz, R.C., J.L. Konkol, J.M. PérezMartínez, and R. Fernandez. 2017b. Management of laurel wilt of avocado, caused by Raffaelea lauricola. Eur. J. Plant Pathol. 149(1):133-143.

Ploetz, R.C., J.M. Pérez-Martínez, E.A. Evans, and S.A. Inch. 2011. Toward fungicidal management of laurel wilt of avocado. Plant Dis. 95(8):977-982.

Savidge, J.A., J.W. Stanford, R.N. Reed, G.R. Haddock, and A.A.Y. Adams. 2011. Canine detection of free-ranging brown tree snakes on Guam. N. Z. J. Ecol. 35(2):174-181.

Waggoner, P.L., M.H. Jones, M. Williams, J.M. Johnston, C.C. Edge, and J.A. Petrousky. 1998. Effects of extraneous odors on canine detection. Proc. Soc. Photographic Instrumentation Eng. 3575: 355-362. 